\title{
Buckle Driven Delamination in Thin Hard Film Compliant Substrate Systems
}

\author{
N. R. Moody ${ }^{1}$, E. D. Reedy, Jr. ${ }^{2}$, E. Corona ${ }^{2}$, D. P. Adams ${ }^{2}$, M. S. Kennedy ${ }^{3}$, M. J. Cordill ${ }^{4}$, D. F. Bahr ${ }^{5}$ \\ 1 Sandia National Laboratories, Livermore, CA 94550 USA \\ 2 Sandia National Laboratories, Albuquerque, NM 87158 USA \\ 3 Clemson University, Clemson, SC 29634 USA \\ 4 Erich Schmid Institute, University of Leoben, A-8700 Leoben, Austria \\ 5 Washington State University, Pullman, WA 99164 USA
}

\begin{abstract}
Deformation and fracture of thin films on compliant substrates are key factors constraining the performance of emerging flexible substrate devices. [1-3] These systems often contain layers of thin polymer, ceramic and metallic films and stretchable interconnects where differing properties induce high normal and shear stresses. [4] As long as the films remain bonded to the substrates, they may deform far beyond their freestanding form. Once debonded, substrate constraint disappears leading to film failure.

[3] Experimentally it is very difficult to measure properties in these systems at sub-micron and nanoscales. Theoretically it is very difficult to determine the contributions from the films, interfaces, and substrates. As a result our understanding of deformation and fracture behavior in compliant substrate systems is limited. This motivated a study of buckle driven delamination of thin hard tungsten films on pure PMMA substrates.

The films were sputter deposited to thicknesses of $100 \mathrm{~nm}, 200 \mathrm{~nm}$, and $400 \mathrm{~nm}$ with a residual compressive stress of $1.7 \mathrm{GPa}$. An aluminum oxide interlayer was added on several samples to alter interfacial composition. Buckles formed spontaneously on the PMMA substrates following film deposition. On films without the aluminum oxide interlayer, an extensive network of small telephone cord buckles formed following deposition, interspersed with regions of larger telephone cord buckles. (Figure 1) On films with an aluminum oxide interlayer, telephone cord buckles formed creating a uniform widely spaced pattern. Through-substrate optical observations revealed matching buckle patterns along the film-substrate interface indicating that delamination occurred for large and small buckles with and without an interlayer. The coexistence of large and small buckles on the same substrate led to two distinct behaviors as shown in Figure 2 where normalized buckle heights are plotted against normalized film stress. The behaviors deviate significantly from behavior predicted by rigid elastic solutions.

To address this issue we developed a finite element analysis technique that employed a cohesive zone model to simulate interfacial crack growth. Specifying the traction-separation relationship, cohesive strength, and work of separation along with film thickness, film stress, and film and substrate properties, buckle width and height were determined as a function of interfacial toughness. The simulations indicate that an analysis based on rigid substrate solutions significantly underestimate toughness for prescribed buckle widths: a result consistent with an analysis by Yu and Hutchinson [5] that pieced together a solution based on non-linear plate theory with a solution for the linear film on substrate problem. More importantly, the results defined a lower limiting bound to seemingly disparate buckle deflection data (Figure 2). The
\end{abstract}

This is an Open Access article distributed under the terms of the Creative Commons Attribution-Noncommercial License 3.0, which permits unrestricted use, distribution, and reproduction in any noncommercial medium, provided the original work is properly cited. 
variance from linear elastic behavior, especially for the small buckles, indicates more than
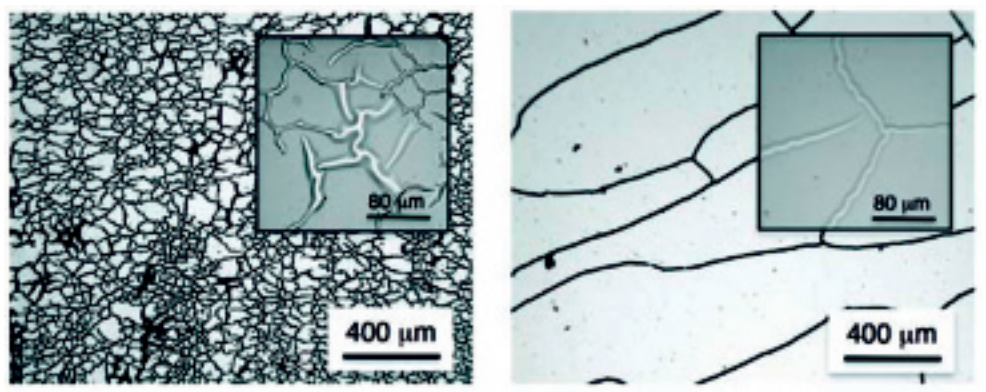

Figure 1. Small telephone cord blisters formed ring-like patterns in $100 \mathrm{~nm}$ thick films deposited on pure PMMA without aluminum oxide interlayers and (b) lattice-like patterns when deposited on substrates with interlayers.

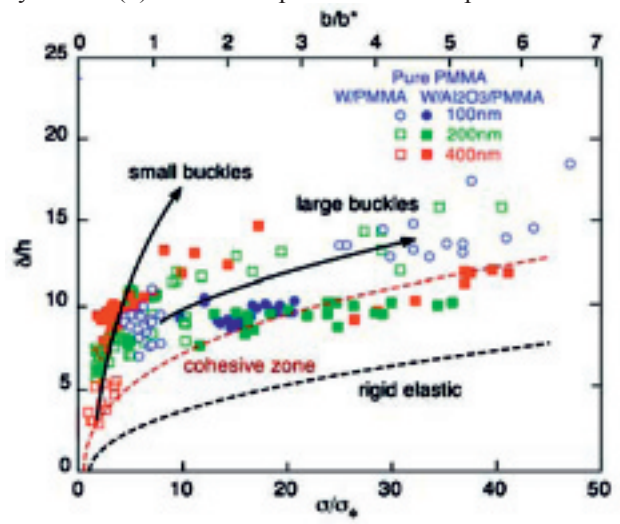

Figure 2. Large and small buckles led to two distinct behaviors where normalized buckle heights are plotted against normalized film stress [4]. The behaviors deviate significantly from behavior predicted by rigid elastic solutions. Cohesive zone model solutions defined a lower limiting bound to buckle deflection data.

substrate compliance is controlling behavior. Comparison of the experimental results with cohesive zone simulations suggests that the two buckle behaviors are associated with different levels of substrate yielding. In this presentation we will use the results to show how substrate compliance and deformation affect delamination and buckling of films on compliant substrates and provide a means to predict device performance.

\section{References}

1. Electronics on Unconventional Substrates--Electrotextiles and Giant-Area Flexible Circuits, M. S. Shur, P. M. Wilson, D. Urban, eds., MRS, 736, (2003).

2. T. Li, Z. Suo, International Journal of Solids and Structure, 44, 1696 (2007).

3. T. Li, Z.Y. Huang, Z.C. Xi, S. P. Lacour, S. Wagner, Z. Suo, Mech. of Mater., 37, 261 (2005).

4. J. W. Hutchinson, Z. Suo, Advances in Applied Mechanics, 29, 63 (1992).

5. H-H. Yu, J. W. Hutchinson, International Journal of Fracture, 113, 39 (2002).

\section{Acknowledgements}

This work is supported by Sandia National Laboratories, a multiprogram laboratory operated by Sandia Corporation, a Lockheed Martin Company for the United States Department of Energy's National Nuclear Security Administration under contract DE-AC04-94AL85000. 